\title{
Awareness of vaccination status and its predictors among working people in Switzerland Chung-Yol Lee ${ }^{1}$, Claudine Naguel${ }^{2}$, Danielle Gyurech ${ }^{3}$, Nicole Duvoisin ${ }^{2}$ and Julian Schilling*2
}

Address: ${ }^{1}$ Now at Bundesamt für Gesundheit, Abteilung Sucht und Aids, Hess-Strasse 27e, CH-3097, Liebefeld, Switzerland, ${ }^{2}$ Institute of Social and Preventive Medicine, University of Zurich, Sumatrastrasse 30, 8006 Zurich, Switzerland and ${ }^{3}$ Travel Clinic, Forchstrasse 92,8008 Zurich, Switzerland

Email: Chung-Yol Lee - chung-yol.lee@bag.admin.ch; Claudine Naguel - nac@meteoswiss.ch;

Danielle Gyurech - reisemedizin@compuserve.com; Nicole Duvoisin - nicoled@ifspm.unizh.ch; Julian Schilling* - juli@ifspm.unizh.ch

* Corresponding author

Published: 2 June 2003

BMC Public Health 2003, 3:18
Received: 23 September 2002

Accepted: 2 June 2003

This article is available from: http://www.biomedcentral.com/I47I-2458/3//8

(c) 2003 Lee et al; licensee BioMed Central Ltd. This is an Open Access article: verbatim copying and redistribution of this article are permitted in all media for any purpose, provided this notice is preserved along with the article's original URL.

\begin{abstract}
Background: Adult vaccination status may be difficult to obtain, often requiring providers to rely on individual patient recall. To determine vaccination status awareness and the sociodemographic predictors of awareness for tetanus, hepatitis $A$ and $B$, tick born encephalitis (TBE) and influenza vaccination.
\end{abstract}

Methods: Multivariate analyses were used to evaluate a questionnaire survey of 10321 employees (4070 women and 625I men aged 15-72 years) of two companies in Switzerland.

Results: Among 10321 respondents, $75.5 \%$ reported knowing their tetanus vaccination status, 64.1\% hepatitis A, 6I.1\% hepatitis B, 64.3\% TBE and 7I.9\% influenza. Between I in 4 and I in 3 employees were not aware of their vaccination status. Differences in awareness for the five vaccinations considered correlated with gender and language. These differences persisted in multivariate analyses.

Conclusion: Women employees, German-speaking employees and employees who paid more attention to their diet were more often aware of their vaccination status. A more reliable and readily accessible data source for vaccination status is needed in order to capitalize on opportunities to update vaccinations among Swiss employees.

\section{Background}

Thanks to efficacious vaccination campaigns, high hygienic standards and a high standard of living, diseases such as poliomyelitis, tetanus, diphtheria, pertussis, and congenital rubella syndrome are now rare in Switzerland [1]. However, the low incidence of these diseases combined with concerns over safety of vaccines have created much controversy [2-9]. The Swiss Federal Office of Public Health (SFOPH) has launched several public awareness campaigns in recent years emphasising the importance of vaccination. Public awareness campaigns are likely to be particularly effective at increasing vaccination rates against diseases occurring seasonally, such as influenza or tick born encephalitis, that require annual vaccination 
campaigns. Vaccines for other diseases, such as hepatitis A and B or tetanus, are not repeated annually. Therefore, vaccination status, including completion of a basic series and last booster dose, can be difficult to obtain. Consequently, health care providers administering vaccinations where medical records are lacking must rely on individual patient recall.

Validation studies of self-reported vaccination status showed that patient self-report was a highly sensitive and moderately specific measure of vaccination status $[10,11]$. One study showed that patient self-report of vaccinations not requiring annual boosters, such as pneumococcal vaccination, had lower specificity than that of vaccinations given annually, such as influenza [11]. More specifically, the study found that although relatively few false-negative cases would have been missed through self-report for both vaccinations $(0-2 \%$ for influenza vaccination and $3-10 \%$ for pneumococcal vaccination, respectively), depending on the population studied, (i.e. veterans administration versus managed care organization), falsepositive cases would have been much more frequent. 21$29 \%$ of reported influenza vaccinations and $46-47 \%$ of reported pneumococcal vaccinations would have been false positive, leading to many missed opportunities to vaccinate [11].

Because people either misplace or neglect to carry their vaccination cards with them, and increasing mobility necessitating periodic changes in health care provider for vaccinations, reliance on self-report will continue to occur, unless new strategies, such as national registries, become standard. But for the time being, such national registries have been shown to have low acceptance [12].

Many vaccine-preventable diseases occur among adults and cause mortality in Europe as well as in the US [1317]. Vaccination screening and administration at the workplace have been shown to be an effective way to deliver comprehensive immunization services to employees [18].

In order to determine the awareness of vaccination status among Swiss employees, we analyzed data on immunization status from the "Check Bus" Project [19], along with demographic, psychological and behavioral predictors of vaccination status awareness.

\section{Review of the studied vaccinations \\ Tetanus}

All men aged 18 to 20 years without a valid tetanus vaccination at the beginning of their compulsory military service in Switzerland are inoculated. The tetanus vaccination was first available in 1938 (Wiedenmann, M., August 2000, Berna, personal communication), and in 1963, the first written recommendation was made (Roost, H.-P., August 2000, SFOPH, personal communication). Currently, tetanus vaccination is recommended for every infant, three times in infancy and three times in childhood. Subsequent routine doses are administered every 10 years unless an injury necessitates an earlier booster. In $1998,93.3 \%$ of Swiss children had completed the tetanus immunization series (three doses) [20]. There were, however, very few tetanus cases registered: between 1 and 5 cases per year from 1988 to 1998 [21], and 4 cases in the year ending August 22, 2000, annual incidence: 0.06/ 100'000 population [22]. By comparison, in 1996 there were 17 reported cases in Germany, annual incidence: $0.2 / 1$ '000'000 population [23] and 8 in the United Kingdom, annual incidence: 0.1/1'000'000 population [3].

\section{Hepatitis $B$}

In 1980, the hepatitis B vaccine was introduced in Switzerland for people at high risk, e.g. health care and dentistry workers, laboratory workers and drug addicts (Wiedenmann, M., August 2000, Berna). Since 1997 hepatitis B has been part of the national immunization program for children between 11 and 15 years of age. Vaccination of newborns is only recommended for groups at risk, e.g. babies with HB-antigen positive mothers [24].

\section{Hepatitis $A$}

In 1992, the active hepatitis A vaccine was introduced in Switzerland. The annual mortality rate for hepatitis A is very low (0.96/1'000'000 population) [25]. The vaccine is mostly administered to people travelling to potentially endemic countries with poor hygiene [26]. Since 1995, the 250 - 500 new cases of infections per year in Switzerland [21] were mostly travellers returning from endemic areas and drug-addicts [27].

\section{Tick Born Encephalitis (TBE)}

TBE is an endemic disease contracted through bites from infected ticks in well-known areas (mostly in Germanspeaking areas of Switzerland). With 68 incident cases in 1998, 112 cases in 1999, 93 cases for 2000 and 107 cases in $2001[28,29]$ and an average overall incidence of $0.46 /$ 1'000'000 population per year, it is a rather rare, but present disease [30]. An active vaccine has been commercially available since 1979 [31]. This vaccine, which is administered as a three dose regimen, is mainly given to people at high risk, i.e. people who regularly spend time in forests in an endemic area, be it for work, or leisure [30].

\section{Influenza}

According to Swiss vaccine recommendations, [32] influenza vaccinations should be performed annually between mid-October and mid-November. The vaccine remains effective for 4-6 months [32]. Current Swiss 
recommendations for influenza vaccination (2001/2002) include persons older than 65 , children and adults with chronic conditions such as heart or lung diseases, as well as those who need regular medical treatment or hospitalizations [33]. For these high-risk groups, the costs are borne by health insurance. While for others, who are also at increased risk or who may risk transmitting the influenza virus to individuals at high risk, such as clinical staff personnel and close contacts of individuals at high risk, insurance has no obligation to bear the costs [33]. Casefatality rates for influenza begin to rise at the age of 45 years and are highest in people with multiple chronic medical conditions [34].

The annual national vaccination campaigns put on by the Swiss government have not been as successful as some had hoped. In 1996, only 3.3\% of all employees had been vaccinated. In that same year, the overall vaccination rate of the Swiss population, using units of sold vaccines, was estimated to be around $6 \%$. It increased to approximately $14 \%$ in 2000 [35]. This vaccination rate is comparable with other European countries. Spain, France, the Netherlands and Italy reached immunization rates of more than $15 \%$ in 1997, while Germany, Great Britain and Belgium have rates ranging from $10-15 \%[35,36]$.

\section{Methods}

The national information and prevention campaign "Check Bus", which took place from August 1996 to August 1998, invited all employees of two nationwide companies (a large Swiss bank and an industrial company) to participate. Participants completed a self-administered questionnaire containing, among others, questions concerning their vaccination status. The questionnaire was available in the three Swiss national languages: German, French and Italian. Participants were asked to present their vaccination certificates. Methods have been described in detail elsewhere [19].

We assessed the status of the following vaccinations: tetanus, hepatitis A and B, tick-borne encephalitis (TBE) and influenza. Influenza vaccination rates were only analyzed for the years 1996 and 1997 as the vaccination period begins in October and the "Check Bus" Project ended in August 1998.

First, we analyzed the following groups of variables for statistical significance in bivariate tests using Chi-square for each vaccine: Demographics (age, gender, marital status, language), psychological factors (general well-being, emotional well-being, stress), and behavioral factors (medication use, alcohol use, smoking status, physical activity and dietary attitudes). Age was categorized into the following groups: $-24,25-34,35-44,45-54$ and 55+ years. We divided marital status into the categories single, married, divorced and widowed. Language represented the language for which the questionnaire was requested and included German, French and Italian. To assess general well-being, the following question was asked: "How is your current state of health?" The respondents had the choice among four answers, ranging from "poor" to "excellent". Emotional well-being was a composite measure of four questions on feeling down or depressed, calm and balanced, tense and nervous, and full of energy and optimism, that evaluated on how many days of the last week respondents felt that way. The four choices were almost every day, on 3-4 days, on 1-2 days and never. The respondents' summary scores were calculated and classified into four groups: poor, moderate, good, and excellent.

Stress was assessed by asking six questions on conditions at work that could be perceived as stressful, such as long working hours, conflicting private life and work, and work under time pressure. Answers were given on a six point Likert scale, summary scores were calculated and following groups defined: never, sometimes, frequent, constant. Medication use assessed the frequency of four common medications, sleeping pills, painkillers, tranquilizers and laxatives. The answers on a five point Likert scale were summed up to a single score and then divided into the groups never, sometimes, frequently and constantly. Alcohol use was largely a measure of frequency and asked the "usual" consumption of alcoholic beverages per week. Answers ranged from never / abstinent to 3 times daily or more often and were categorised into never/seldom, once/ several times a week, once/ several times a day.

Smoking status was divided into current smoker (ever smoked regularly during more than 6 months and currently smoking), ex-smoker (ever smoked regularly during more than 6 months, but not currently smoking) and non-smoker (never smoked regularly for more than 6 months and not currently smoking). We accepted the possibility that some of the participants would not fall into any of the three categories by choosing a relatively long duration of regular smoking in favour of exclusion of experimenters and occasional smokers. Like alcohol use, physical activity assessed mainly the frequency by asking how many days per week the participant was involved in any leisure activity through which he or she would sweat ("e.g. brisk walking, running, bicycling, etc."), ranging from never to $7 \mathrm{x}$ per week. Finally, dietary attitude was assessed by asking about any particular attention participants paid to twelve components, such as sweets, salt, fat, alcohol, diversity of food, regular meals, vegetarian diet, etc. A summary score was calculated from dichotomous answers on each component and then categorised into following groups: paid strict attention, paid attention, paid little attention, paid no attention. 
Table I: Bivariate Analyses

\begin{tabular}{|c|c|c|c|c|c|c|c|c|c|c|c|c|c|}
\hline & Age & Gender & Language & $\begin{array}{c}\text { Marital } \\
\text { Status }\end{array}$ & $\begin{array}{l}\text { Hierarchy } \\
\text { at Work }\end{array}$ & $\begin{array}{l}\text { Stress at } \\
\text { Work }\end{array}$ & $\begin{array}{r}\text { General } \\
\text { Well-being }\end{array}$ & $\begin{array}{r}\text { Emotional } \\
\text { Well-being }\end{array}$ & Smoking & $\begin{array}{r}\text { Alcohol } \\
\text { Use }\end{array}$ & $\begin{array}{r}\text { Medication } \\
\text { Use }\end{array}$ & $\begin{array}{r}\text { Dietary } \\
\text { Attitudes }\end{array}$ & $\begin{array}{l}\text { Physical } \\
\text { Activity }\end{array}$ \\
\hline Tetanus & $<0.001$ & $<0.001$ & $<0.001$ & $<0.001$ & 0.986 & $<0.001$ & $<0.001$ & $<0.001$ & $<0.001$ & $<0.001$ & 0.006 & $<0.001$ & $<0.001$ \\
\hline Hepatitis B & 0.445 & $<0.001$ & $<0.001$ & 0.376 & 0.222 & 0.003 & 0.058 & 0.032 & $<0.001$ & $<0.001$ & 0.725 & $<0.001$ & 0.294 \\
\hline Hepatitis A & 0.128 & $<0.001$ & $<0.001$ & 0.017 & 0.528 & $<0.001$ & 0.002 & 0.028 & $<0.001$ & $<0.001$ & 0.745 & $<0.001$ & 0.115 \\
\hline $\begin{array}{l}\text { Tick born } \\
\text { Encephalitis }\end{array}$ & 0.980 & $<0.001$ & $<0.001$ & 0.457 & 0.041 & 0.002 & 0.008 & 0.186 & $<0.001$ & $<0.001$ & 0.345 & $<0.001$ & 0.157 \\
\hline Influenza & $<0.001$ & $<0.001$ & $<0.001$ & 0.529 & 0.094 & 0.005 & 0.092 & 0.648 & $<0.001$ & 0.002 & 0.111 & $<0.001$ & 0.472 \\
\hline
\end{tabular}

all categorical (Chi-squared test)

Variables with statistically significant differences in bivariate tests (Chi-squared test) at $\mathrm{p}<0.05$ were entered into logistic regression models using SPSS 10. A two-tailed significance level was set at $\mathrm{p}<0.01$ for the multivariate tests.

\section{Results}

A total of 10321 (41\% of all employees) data sets were available for analysis. Respondents were $39.4 \%$ ( $\mathrm{n}=$ 4070) female and $60.6 \%(n=6251)$ male, corresponding with the gender distribution in the participating companies. Of the participants, $82.3 \%(\mathrm{n}=8495)$ were Germanspeaking, $13.8 \%(\mathrm{n}=1419)$ French-speaking and 3.9\% (n $=407$ ) Italian-speaking. At the time of questioning $83.9 \%$ $(\mathrm{n}=8657)$ worked for the bank and $16.1 \%(\mathrm{n}=1664)$ for the industrial company. Of the participants, $67.2 \%(\mathrm{n}=$ $6938)$ were staff, $23.1 \%(\mathrm{n}=2382)$ were cadre, $7.4 \%(\mathrm{n}=$ $767)$ were supervisors, and $2.3 \%(n=234)$ withheld this information.

\section{Tetanus}

Of the 10063 participants who answered the questions on tetanus vaccination ( 258 or $2.5 \%$ did not), $75.5 \%$ (7599) knew their vaccination status (vaccinated 7446 or $74.0 \%$, never vaccinated 153 or $1.5 \%$ ) and $24.5 \%$ (2464) did not (the difference relative to $100 \%$ is due to missing answers). (Figure 1)

Bivariate analyses (Table 1) showed that age, gender, language, marital status, stress at work, emotional well-being, general well-being, smoking status, alcohol use, dietary attitude, physical activity (all p < 0.001) and medication use $(\mathrm{p}=0.006)$ were all associated with awareness of tetanus vaccination status, while only employment hierarchy was not.

In the logistic regression model, four of the significant variables in the bivariate analyses remained independently associated with awareness of tetanus vaccination status: age, gender, language and smoking, while marital status, stress at work, general well-being, emotional well-being, alcohol use, medication use, dietary attitudes and physical activity did not.

\section{Hepatitis B}

Of the 9427 participants who answered the questions on hepatitis B vaccination ( 894 or $8.7 \%$ did not), $61.1 \%$ (5757) knew their vaccination status (vaccinated 299 or $3.2 \%$, not vaccinated 5458 or $57.9 \%$ ), while $38.9 \%$ (3670) did not. Bivariate analyses revealed gender, language, smoking status, alcohol use, dietary attitudes (all $\mathrm{p}$ $<0.001)$, stress $(\mathrm{p}=0.003)$ and emotional well-being ( $\mathrm{p}$ $=0.032$ ) to be associated with awareness of hepatitis $\mathrm{B}$ vaccination status, while age, marital status, employment hierarchy, general well-being, medication use and physical activity were not.

In the logistic regression model, three variables were independently associated with awareness of hepatitis B vaccination status: gender, language and dietary attitudes.

\section{Hepatitis A}

Among 9383 respondents who answered the questions on hepatitis A vaccination (938 or $9.1 \%$ did not), $64.1 \%$ (6011) knew their vaccination status (vaccinated 1574 or $16.8 \%$, not vaccinated 4437 or $47.3 \%$ ), while $35.9 \%$ (3372) did not.

Bivariate analyses showed awareness of hepatitis A vaccination status to be associated with the following variables: gender, language, stress, smoking status, alcohol use, diet (all $\mathrm{p}<0.001)$, general well-being $(\mathrm{p}=0.002)$, emotional well-being $(\mathrm{p}=0.028)$ and marital status $(\mathrm{p}=0.017)$, while age, employment hierarchy, medication use and physical activity were not. In the logistic regression model, four variables were independently associated with awareness of hepatitis A vaccination status: gender, language, smoking status and dietary attitudes.

\section{Tick-Borne Encephalitis}

Among 9520 respondents who answered the questions on tick-borne encephalitis ( 801 or $7.8 \%$ did not), $64.3 \%$ (6122) knew their vaccination status (vaccinated 224 or $2.4 \%$, not vaccinated 5898 or $62.0 \%$ ), while $35.7 \%$ (3398) did not. 


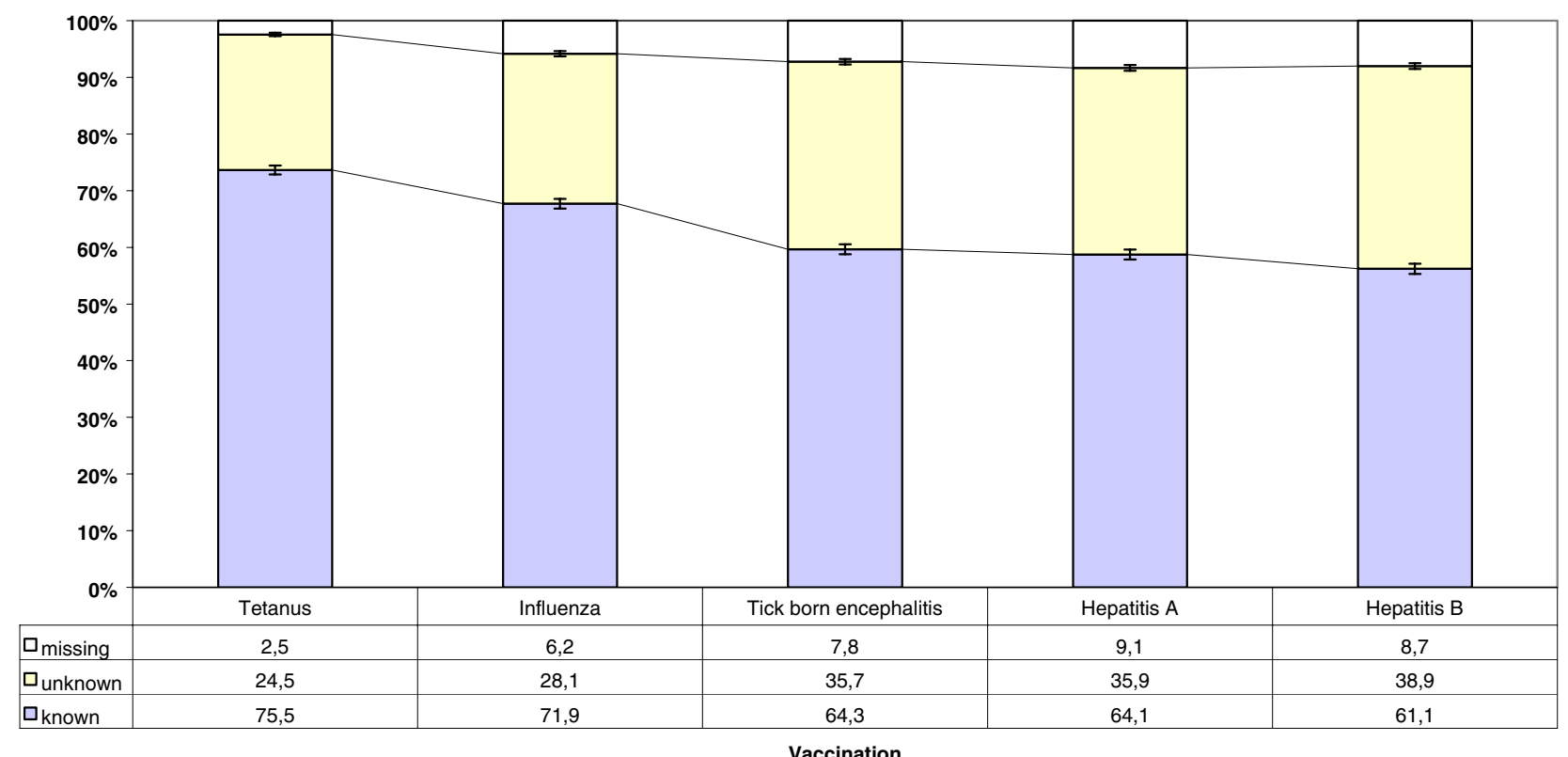

\section{Figure I}

Participants' awareness of vaccination status for the different vaccinations $\left(v^{2}-\right.$ Test $\left.p<0.00 \mathrm{I}\right)$. The $95 \% \mathrm{Cl}$ for each proportion value was below $1 \%$.

Bivariate analyses showed awareness of TBE vaccination status to be associated with the following variables: gender, language, smoking status, alcohol use, diet (all $\mathrm{p}<$ $0.001)$, stress level (0.002), general well-being (0.008) and employment hierarchy (0.041), while not associated with age, marital status, emotional well-being, medication use and physical activity.

In the logistic regression model, five variables were independently associated with awareness of TBE vaccination status: gender, language, employment hierarchy, alcohol use and dietary attitudes.

\section{Influenza}

Among 9679 respondents who answered the questions on influenza (642 or $6.2 \%$ did not), 71.9\% (6122) knew their vaccination status for 1996 and 1997 (vaccinated 308 or $3.2 \%$, not vaccinated 6650 or $68.7 \%, 1996$ vaccinated 204 or $2.1 \%, 1997$ vaccinated 104 or $1.1 \%$ ), while $28.1 \%$ (2718) did not.

Bivariate analyses showed awareness of 1996 and 1997 influenza vaccination status to be associated with the following variables: age, gender, language, smoking status, diet (all p < 0.001), stress (0.005), and alcohol use (0.002), while marital status, employment hierarchy, gen- eral well-being, emotional well-being, medication use and physical activity were not.

In the logistic regression model, six variables were independently associated with awareness of influenza vaccination status in 1996 and 1997: age, gender, language, stress, smoking status, and dietary attitudes.

Table 2 shows the respondents awareness of vaccination status (number of individuals with vaccination status known/unknown) for the characteristic variables together with the results of the overall logistic regression for each vaccination.

\section{Discussion}

In this working population of two large firms in Switzerland, the proportion of known vaccination status (vaccinated or not vaccinated) for five different vaccinations varied between $61.1 \%$ for hepatitis B and $75.5 \%$ for tetanus (Figure 1).

The proportion of employees vaccinated against tetanus $(74.0 \%)$ was almost identical with a previous study among primary care physicians and their employees in Switzerland, where $74.2 \%$ of the medical practice employees stated to have been vaccinated against tetanus [37]. 
Table 2: Logistic Regression

\begin{tabular}{|c|c|c|c|c|c|c|c|c|c|c|}
\hline & \multicolumn{2}{|c|}{ Tetanus } & \multicolumn{2}{|c|}{ Hepatitis B } & \multicolumn{2}{|c|}{ Hepatitis A } & \multicolumn{2}{|c|}{$\begin{array}{c}\text { Tick Born } \\
\text { Encephalitis }\end{array}$} & \multicolumn{2}{|c|}{ Influenza } \\
\hline & $\mathrm{n}$ & $\mathrm{OR}(\mathrm{Cl})$ & $\mathrm{n}$ & $\mathrm{OR}(\mathrm{Cl})$ & $N$ & $\mathrm{OR}(\mathrm{Cl})$ & $N$ & $\mathrm{OR}(\mathrm{Cl})$ & $\mathrm{n}$ & $\mathrm{OR}(\mathrm{Cl})$ \\
\hline \multicolumn{11}{|l|}{ Age } \\
\hline-24 & $807 / 153$ & 1.0 & $573 / 338$ & - & $584 / 330$ & - & $575 / 330$ & - & $638 / 278$ & 1.0 \\
\hline $25-34$ & $2520 / 600$ & $\begin{array}{r}0.84 \\
(0.65,1.00)\end{array}$ & $1781 / 1118$ & - & $1912 / 986$ & - & $1900 / 1052$ & - & $2211 / 772$ & $\begin{array}{r}1.22 \\
(1.04,1.45)\end{array}$ \\
\hline $35-44$ & $2012 / 619$ & $\begin{array}{r}0.67 \\
(0.53,0.84)\end{array}$ & $1496 / 935$ & - & $1510 / 869$ & - & $1589 / 868$ & - & $1859 / 660$ & $\begin{array}{r}1.24 \\
(1.04,1.47)\end{array}$ \\
\hline $45-54$ & $1647 / 760$ & $\begin{array}{r}0.48 \\
(0.38,0.61)\end{array}$ & $1373 / 915$ & - & $1438 / 850$ & - & |478/828 & - & $162 \mid / 729$ & $\begin{array}{r}0.97 \\
(0.81,1.15)\end{array}$ \\
\hline $55+$ & $613 / 332$ & $\begin{array}{r}0.39 \\
(0.30,0.52)\end{array}$ & $534 / 364$ & - & $567 / 337$ & - & $580 / 320$ & - & $632 / 279$ & $\begin{array}{r}0.99 \\
(0.80,1.23)\end{array}$ \\
\hline \multicolumn{11}{|l|}{ Gender } \\
\hline Female & $307 \mid / 885$ & 1.0 & $2368 / 1293$ & 1.0 & $2510 / 1177$ & 1.0 & $2521 / 1198$ & 1.0 & $2865 / 922$ & 1.0 \\
\hline Male & $4528 / 1579$ & $\begin{array}{r}0.84 \\
(0.75,0.95)\end{array}$ & $3389 / 2377$ & $\begin{array}{r}0.79 \\
(0.72,0.86)\end{array}$ & $3501 / 2195$ & $\begin{array}{r}0.77 \\
(0.69,0.85)\end{array}$ & $3601 / 2200$ & $\begin{array}{r}0.7 \\
(0.65,0.79)\end{array}$ & $4096 / 1796$ & $\begin{array}{r}0.73 \\
(0.81,1.23)\end{array}$ \\
\hline \multicolumn{11}{|l|}{ Language } \\
\hline German & $6405 / 1887$ & 1.0 & $4889 / 2922$ & 1.0 & $5048 / 2706$ & 1.0 & $5319 / 2580$ & 1.0 & $5831 / 2164$ & 1.0 \\
\hline French & $962 / 411$ & $\begin{array}{r}0.71 \\
(0.62,0.82)\end{array}$ & $605 / 615$ & $\begin{array}{r}0.61 \\
(0.54,0.69)\end{array}$ & 698/539 & $\begin{array}{r}0.72 \\
(0.63,0.82)\end{array}$ & $544 / 679$ & $\begin{array}{r}0.39 \\
(0.34,0.45)\end{array}$ & $824 / 461$ & $\begin{array}{r}0.67 \\
(0.66,0.81)\end{array}$ \\
\hline Italian & $232 / 166$ & $\begin{array}{r}0.41 \\
(0.32,0.5 I)\end{array}$ & $263 / 133$ & $\begin{array}{r}1.17 \\
(0.94,1.46)\end{array}$ & $265 / 127$ & $\begin{array}{r}1.15 \\
(0.92,0.14)\end{array}$ & $259 / 139$ & $\begin{array}{r}0.92 \\
(0.74,1.14)\end{array}$ & $306 / 93$ & \\
\hline \multicolumn{11}{|c|}{ Hierarchy at Work } \\
\hline Staff & $5099 / 1663$ & - & $3876 / 2483$ & - & $4058 / 2301$ & - & $4088 / 2338$ & 1.0 & $464 I / / 875$ & - \\
\hline Superiors & $559 / 180$ & - & $450 / 254$ & - & $454 / 234$ & - & $477 / 226$ & $\begin{array}{r}1.39 \\
(1.16,1.66)\end{array}$ & $534 / 182$ & - \\
\hline Cadre & $1756 / 575$ & - & $1300 / 857$ & - & $1365 / 769$ & - & $1430 / 755$ & $\begin{array}{r}1.23 \\
(1.10,1.37)\end{array}$ & $1626 / 610$ & - \\
\hline \multicolumn{11}{|c|}{ Stress at Work } \\
\hline never & $120 / 73$ & 1.0 & $88 / 95$ & 1.0 & $91 / 92$ & 1.0 & $95 / 87$ & 1.0 & || $5 / 7 \mid$ & 1.0 \\
\hline sometimes & $|888 / 66|$ & $\begin{array}{r}1.39 \\
(0.94,2.06)\end{array}$ & $1469 / 909$ & $\begin{array}{r}1.48 \\
(1.05,2.09)\end{array}$ & $1535 / 836$ & $\begin{array}{r}1.56 \\
(1.10,2.20)\end{array}$ & $1565 / 848$ & $\begin{array}{r}1.66 \\
(1.21,2.28)\end{array}$ & $1730 / 7 \mid 5$ & $\begin{array}{r}1.42 \\
(1.04,1.95)\end{array}$ \\
\hline frequent & $5146 / 1578$ & $\begin{array}{r}1.61 \\
(1.09,2.37)\end{array}$ & $3869 / 2446$ & $\begin{array}{r}1.58 \\
(1.13,2.21)\end{array}$ & $4047 / 2240$ & $\begin{array}{r}1.67 \\
(1.18,2.34)\end{array}$ & $2249 / 4126$ & $\begin{array}{r}1.71 \\
(1.25,2.28)\end{array}$ & $4709 / 1773$ & $\begin{array}{r}1.61 \\
(1.18,2.20)\end{array}$ \\
\hline constant & $445 / 152$ & $\begin{array}{r}1.70 \\
(1.09,2.63)\end{array}$ & $331 / 220$ & $\begin{array}{r}1.68 \\
(1.15,2.47)\end{array}$ & $338 / 204$ & $\begin{array}{r}1.72 \\
(1.17,2.54)\end{array}$ & $336 / 214$ & $\begin{array}{r}1.70 \\
(1.19,2.43)\end{array}$ & $407 / 159$ & $\begin{array}{r}1.66 \\
(1.16\end{array}$ \\
\hline \multicolumn{11}{|l|}{ Smoking } \\
\hline $\begin{array}{l}\text { Non- } \\
\text { Smoker }\end{array}$ & $3981 / 1103$ & 1.0 & $3013 / 1777$ & 1.0 & $3153 / 1612$ & 1.0 & $3186 / 1632$ & 1.0 & $3616 / 1276$ & 1.0 \\
\hline Smoker & $2347 / 884$ & $\begin{array}{r}0.78 \\
(0.69,0.87)\end{array}$ & $1755 / 1250$ & $\begin{array}{r}0.88 \\
(0.80,0.97)\end{array}$ & $1822 / 1172$ & $\begin{array}{r}0.84 \\
(0.76,0.93)\end{array}$ & $1864 / 1180$ & $\begin{array}{r}0.87 \\
(0.79,0.96)\end{array}$ & $2|24 / 97|$ & $\begin{array}{r}0.80 \\
(0.73,0.89)\end{array}$ \\
\hline Ex-Smoker & $|27| / 477$ & $\begin{array}{r}0.94 \\
(0.81,1.08)\end{array}$ & $989 / 643$ & $\begin{array}{r}0.93 \\
(0.82,1.05)\end{array}$ & $1036 / 588$ & $\begin{array}{r}0.95 \\
(0.84,1.08)\end{array}$ & $1072 / 586$ & $\begin{array}{r}0.97 \\
(0.86,1.10)\end{array}$ & $|22| / 47 \mid$ & $\begin{array}{r}0.98 \\
(0.86,1.12)\end{array}$ \\
\hline \multicolumn{11}{|c|}{ Alcohol Use } \\
\hline $\begin{array}{l}\text { never/ } \\
\text { seldom }\end{array}$ & $2666 / 813$ & 1.0 & $2044 / 1224$ & 1.0 & $2|42 /| 13 \mid$ & 1.0 & $2143 / 1156$ & 1.0 & $2426 / 912$ & - \\
\hline $\begin{array}{l}\text { once/ } \\
\text { several } \\
\text { times a } \\
\text { week }\end{array}$ & $4308 / 1335$ & $\begin{array}{r}1.06 \\
(0.95,1.19)\end{array}$ & $2335 / 2041$ & $\begin{array}{r}1.04 \\
(0.94,1.14)\end{array}$ & $3374 / 1863$ & $\begin{array}{r}1.05 \\
(0.95,1.15)\end{array}$ & $3481 / 1857$ & $\begin{array}{r}1.10 \\
(0.99,1,21)\end{array}$ & $3927 / 1506$ & - \\
\hline $\begin{array}{l}\text { once/ } \\
\text { several } \\
\text { times a day }\end{array}$ & $619 / 315$ & $\begin{array}{r}0.98 \\
(0.81,1.18)\end{array}$ & $472 / 404$ & $\begin{array}{r}0.84 \\
(0.71,0.98)\end{array}$ & $489 / 377$ & $\begin{array}{r}0.85 \\
(0.72,1.00)\end{array}$ & $492 / 384$ & $\begin{array}{r}0.83 \\
(0.70,0.98)\end{array}$ & $602 / 299$ & - \\
\hline \multicolumn{11}{|c|}{ Dietary Attitudes } \\
\hline $\begin{array}{l}\text { paid strict } \\
\text { attention }\end{array}$ & $365 / 115$ & 1.0 & $298 / 136$ & 1.0 & $308 / 132$ & 1.0 & $315 / 129$ & 1.0 & $359 / 97$ & 1.0 \\
\hline
\end{tabular}


Table 2: Logistic Regression (Continued)

\begin{tabular}{|c|c|c|c|c|c|c|c|c|c|c|}
\hline $\begin{array}{l}\text { paid } \\
\text { attention }\end{array}$ & $2246 / 678$ & $\begin{array}{r}0.97 \\
(0.76,1.24)\end{array}$ & $1787 / 968$ & $\begin{array}{r}0.88 \\
(0.70,1.10)\end{array}$ & $1840 / 870$ & $\begin{array}{r}0.96 \\
(0.76,1.20)\end{array}$ & $1910 / 878$ & $\begin{array}{r}0.91 \\
(0.73,1.14)\end{array}$ & $21 / 2 / 732$ & $\begin{array}{r}0.79 \\
(0.62,1.00)\end{array}$ \\
\hline $\begin{array}{l}\text { paid little } \\
\text { attention }\end{array}$ & $2709 / 808$ & $\begin{array}{r}0.88 \\
(0.69,1.13)\end{array}$ & $1995 / 1293$ & $\begin{array}{r}0.76 \\
(0.61,0.94)\end{array}$ & $2102 / 1190$ & $\begin{array}{r}0.81 \\
(0.65,1.02)\end{array}$ & $2152 / 1169$ & $\begin{array}{r}0.80 \\
(0.64,1.00)\end{array}$ & $2442 / 929$ & $\begin{array}{r}0.72 \\
(0.57,0.92)\end{array}$ \\
\hline $\begin{array}{l}\text { paid no } \\
\text { attention }\end{array}$ & $2279 / 863$ & $\begin{array}{r}0.79 \\
(0.61,1.01)\end{array}$ & $1677 / \mid 273$ & $\begin{array}{r}0.70 \\
(0.56,0.87)\end{array}$ & $1761 / 1180$ & $\begin{array}{r}0.74 \\
(0.59,0.92)\end{array}$ & $1745 / 1222$ & $\begin{array}{r}0.70 \\
(0.56,0.88)\end{array}$ & $2048 / 960$ & $\begin{array}{r}0.65 \\
(0.51,0.83)\end{array}$ \\
\hline
\end{tabular}

$\mathrm{n}=$ vaccination status known / unknown, OR = Odds Ratio (coding of dependent variable: unknown $=0$, known $=\mathrm{I}$ ), $\mathrm{Cl}=95 \% \mathrm{Confidence} \mathrm{Interval}$

The proportion of employees in our study population vaccinated against influenza in 1996 or 1997 (3.2\%) corresponded with estimates by the Federal Office of Public Health among employees in 1996 where a vaccination rate of 3.3\% was found. However, this similarity may be misleading, as a validation study for influenza vaccination rates has shown that between $20-30 \%$ of those indicating to have been vaccinated for Influenza actually had not been vaccinated, when checked against data in hospital records [11].

In the bivariate analyses, six independent variables show a significant association with awareness of vaccination status for all five immunizations: gender, language, stress, smoking, alcohol use and dietary behavior.

The results of the logistic regression analyses seem to discriminate mainly between two groups of independent variables. Two variables, gender and language, were associated with all five types of vaccination, while the rest of the independent variables were associated with four (dietary behavior), three (smoking status), two (age) or one type of vaccination (employment hierarchy and stress). Interestingly, alcohol use, which was associated with all five immunizations in bivariate analyses, was only independently associated with one of the five immunizations in regression analyses.

One important weakness of our study is the lack of data on non-participants as we were denied access to this information. This makes it impossible for us to compare participants with non-participants in order to detect any bias due to differential participation. Details concerning representativeness have been discussed in our methods paper [19].

The Check Bus Project was performed during a period of major restructuring in one of the two enterprises involved, which mostly concerned employees in the French-speaking part of Switzerland where the participation rate was consequently lower. This restructuring could have negatively influenced the participation rate. We did not send out reminders and the Check Bus visited the different companies sites only once. In view of this the participation rate of $41 \%$ may be regarded as rather high.

Women, German-speaking people, to a lesser degree people who pay more attention to a healthy diet, and the nonsmokers (as compared to the smokers, but not ex-smokers) seem to be more aware of their vaccination status.

Our results show that between 1 in 4 to 1 in 3 of healthy Swiss employees are unaware of their vaccination status, even for seasonally recommended vaccines. This indicates a need for a more reliable and readily accessible, i.e. electronic, central database where individual vaccination records are stored. More research into the acceptability of such a database among the population and health care providers of Switzerland is also needed.

\section{Competing Interests}

There are no competing interests

\section{Authors' contributions}

CL performed the statistical analyses and participated in the design of the manuscript. CN conceived the study, drafted the manuscript and participated in the statistical analyses, DG participated in the drafting of the manuscript and the study coordination, ND participated in the statistical analyses and the manuscript preparation, and JS participated in the manuscript preparation and study coordination.

\section{Acknowledgements}

We would like to thank the staff of the Check Bus Team for their help in the collection of the data and Jenny Piket for proof-reading the manuscript.

The Check Bus Project was supported by the Credit Suisse Group, Siemens Schweiz AG, Sanitas Health Insurance Company and Europe Assistance.

\section{References}

I. Vranjes N, Gyurech D, Schilling J, Gugelmann R and Steffen R: Durchimpfung von Kleinkindern im Kanton Zürich Schweiz Med Wochenschr 1996, I 26:22-26.

2. Impfschäden: Schreckliche Bestätigung Orizzonti Mitteilungen 2000.

3. Bedford $\mathrm{H}$ and Elliman $\mathrm{D}$ : Concerns about immunisation $B M$ 2000, 320(7229):240-3.

4. Czimmek A: Impfreaktionen Deutsches Journal für Homöopathie 1995. 
5. Radke D: Impfungen Deutsches Journal für Homöopathie 1995.

6. Ascherio A, Zhang SM, Hernan MA, Olek MJ, Coplan PM and Brodovicz K: Hepatitis $B$ vaccination and the risk of multiple sclerosis N Engl J Med 200I, 344(5):327-32.

7. Confavreux C, Suissa S, Saddier P, Bourdes V and Vukusic S: Vaccinations and the risk of relapse in multiple sclerosis. Vaccines in Multiple Sclerosis Study Group N Engl J Med 200I, 344(5):319-26.

8. DeStefano F and Chen RT: Autism and measles-mumps-rubella vaccination: controversy laid to rest? CNS Drugs 200I, I5(II):83I-7.

9. Phelan AT: MMR and autism: an overview of the debate to date $B r J$ Nurs 2002, I I (9):62 I-5

10. Nichol KL, Korn JE and Baum P: Estimation of outpatient risk characteristics and influenza vaccination status: validation of a self-administered questionnaire $A m$ J Prev Med I99I, 7(4): 199203.

II. Mac Donald R, Baken L, Nelson A and Nichol KL: Validation of selfreport of influenza and pneumococcal vaccination status in elderly outpatients American Journal of Preventive Medicine 1999, 16(3): 173-177.

12. Christakis DA, Stewart L, Bibus D, Stout JW, Zerr DM and MacDonald JK: Providers' perceptions of an immunization registry $\mathrm{Am}$ J Prev Med 1999, I7(2): 147-50.

13. Gardner $P$ and Schaffner W: Immunization of adults N Engl] Med 1993, 328(I 7): I252-8.

14. Comprehensive delivery of adult vaccination - Minnesota, 1986-I 992 MMWR Morb Mortal Wkly Rep 1993, 42(39):768-70.

15. Eickhoff TC: Adult immunizations: how are we doing? Hosp Pract (Off Ed) 1996, 3 I(I I): I07-8. III-2, II5-7, passim

16. Williams WW, Hickson MA, Kane MA, Kendal AP, Spika JS and Hinman AR: Immunization policies and vaccine coverage among adults. The risk for missed opportunities Ann Intern Med 1988, 108(4):616-25.

17. Hofmann F: [Vaccination in adults] Ther Umsch 1994, 5 I (8):57782.

18. Grabenstein JD, Smith LJ, Carter DW, Engler RJ, Evans R 3rd and Summers RJ: Comprehensive immunization delivery in conjunction with influenza vaccination Arch Intern Med 1986, I 46(6): I I89-92.

19. Schilling J, Lee C-Y, Faisst K, Dixon M, von Mühlinen I and Itten E: Methods of the National Check Bus Project Sozial- und Präventivmedizin 200I, 46: 195-206.

20. Kleinkinderimpfungen: Repräsentative Erhebung zur Durchimpfung in der Schweiz Bull BAG 2000, 20:356-61.

21. BAG: Meldesystem für Infektionskrankheiten Infektionskrankheiten, Schweiz: BAG 1998.

22. Meldungen Infektionskrankheiten BAG-Bulletin 2000, 35:684685.

23. Zahlen impfpräventabler Krankheiten in der Vor-Impfära Focus Vaccination 1999, I:2.

24. BAG: Empfehlungen zur Hepatitis-B-Impfung, Supplementum II: BAG; 1997.

25. Spuhler T: Kommentar zum Bericht über die Letalität der Hepatitis A in der Schweiz BAG-Bulletin 1997, 38:5.

26. Marchou B, Picot N and Massip P: Vaccinations of the traveller Annales de Médecine Interne 1998, I49(6):332-9.

27. BAG: Infektionskrankheiten in der Schweiz: Bundesamt für Gesundheit; 1998.

28. BAG: Meldesystem für Infektionskrankheiten. Zeckenenzephalitis, Woche 38.: Bundesamt für Gesundheit; 2000.

29. BAG: Meldungen Infektionskrankheiten Berne: Bundesamt fü Gesundheit; 2002, Report No.: 5:

30. Muller A: Active vaccination against early summer meningoencephalitis Schweiz Med Wochenschr 1998, I28(2728): $1110-6$.

31. FSME-Schutz nach Zeckenstich Focus Vaccination 1999, 3.

32. BAG: Empfehlungen zur Grippeprävention Stand: August 2000: Bundesamt für Gesundheit, Arbeitsgruppe Influenza, Schweizerische Kommission für Impffragen; 2000.

33. Müller D: BAG Grippeimpfempfehlung 200 I/2002 BAG-Bulletin 2001 .

34. Zimmerman $R$ : Lowering the age for routine influenza vaccination to $\mathbf{5 0}$ years: AAFP leads the nation in influenza vaccine policy American Family Physician 1999, 60(7):206I-6.
35. Zimmerli $S$ and Mühlemann K: Der aktuelle Stand der Grippeprävention Schweizerische Ärztezeitung 200I, 82(37):|96I1966.

36. BAG: Die Grippe in der Schweiz Unterlagen zur Vernehmlassung der Pandemieverordnung; 2000.

37. Bovier P and Gallacchi MB: Impfungen bei Erwachsenen in der Schweiz: die Meinungen der Grundversorger (Vaccinations among adults in Switzerland: the opinions of primary care physicians) Bulletin des Bundesamtes für Gesundheitswesen 2000, II:220-225.

\section{Pre-publication history}

The pre-publication history for this paper can be accessed here:

http://www.biomedcentral.com/1471-2458/3/18/prepub
Publish with Bio Med Central and every scientist can read your work free of charge

"BioMed Central will be the most significant development for disseminating the results of biomedical research in our lifetime. "

Sir Paul Nurse, Cancer Research UK

Your research papers will be:

- available free of charge to the entire biomedical community

- peer reviewed and published immediately upon acceptance

- cited in PubMed and archived on PubMed Central

- yours - you keep the copyright
BioMedcentral 